\title{
A hybrid approach for fault diagnosis of planetary bearings using an internal vibration sensor
}

\author{
Zhiqi Fan ${ }^{1}$, Huaizhong $\mathrm{Li}^{2 *}$ \\ ${ }^{1}$ School of Mechanical and Manufacturing Engineering, The University of New South \\ Wales, Sydney, NSW 2052, Australia \\ ${ }^{2}$ Griffith School of Engineering, Gold Coast Campus, Griffith University, QLD 4222,
} Australia

\begin{abstract}
Planetary gearboxes exhibit unique challenges in bearing fault detection.
This paper presents a hybrid approach for fault diagnosis of planetary bearings using an internal vibration sensor and novel signal processing strategies. An accelerometer is mounted internally on the planet carrier to address the issues of variable transmission path. An effective bearing faults detection algorism is developed by employing several advanced signal processing techniques, including Cepstrum whitening, minimum entropy deconvolution (MED), spectral kurtosis (SK) and envelope analysis. The adverse effect of the electromagnetic interference in the signal due to the use of a slip ring is tackled by optimizing the SK technique for demodulation band selection. The proposed method is assessed by analyzing experimental data from a planetary gearbox test rig with seeded bearing faults. The
\end{abstract}

\footnotetext{
* Corresponding author: Dr Huaizhong Li. Griffith School of Engineering, Gold Coast Campus, Griffith University, QLD 4222, Australia. Phone: +61 (0) 7555 28252; Telefax: +61 (0) 7555 28065; E-mail: lihuaizhong@gmail.com; h.li@griffith.edu.au
} 
result shows that the new method can effectively detect both inner race and outer race faults of the planetary bearing.

Keywords: Fault diagnosis; planetary gearbox; bearing; signal processing; vibration condition monitoring

\section{Introduction}

With the benefits of strong load-bearing capacity, compact, light weight and large transmission ratio, planetary gearboxes are widely applied in the fields of automobiles, helicopters, wind turbines, etc. [1]. Due to the harsh working conditions, some key components of planetary gearboxes, such as gears and bearings, are extremely vulnerable to faults after long-time operation. The failure of gears or bearings may cause the breakdown of the entire system[2]. Effective fault diagnosis methods are needed for preventing accidents and reducing the maintenance costs. However, planetary gearboxes exhibit unique challenges in bearing fault detection. They comprise many rotating components which lead to contamination by strong background noise. The transmission path between an externally mounted sensor and fault signal source is time varying. Thus traditional fault diagnosis methods developed for fixed-axis gearboxes may not work for planetary gearboxes.

There has been extensive research on condition monitoring and fault diagnosis of planetary gearboxes. McFadden and Howard[3] applied the technique of time 
synchronous averaging (TSA) for diagnosing the local defects in the planet gear and the sun gear. Antoni and Randall proposed a method called discrete random separation (DRS) [4] for the separation of gear signals and faulty bearing signals in planetary gearbox, in which the incipient bearing failure can be detected after the removal of deterministic gear signals. Feng et al. [5] presented a joint amplitude and frequency demodulation method for gear faults diagnosis of planetary gearbox. In [6], the methods of ensemble empirical mode decomposition (EEMD) and energy separation were combined by Feng et al. for detecting the sun gear faults of planetary gearbox. Barszcz and Randall [7] applied the technique of spectral kurtosis for the ring gear crack detection of a planetary gearbox. Yu et al. [8] used the continuous wavelet transform (CWT) or discrete wavelet transform (DWT) on the TSA signal for further optimizing the planet gear diagnosis. Lei et al.[9] proposed an adaptive stochastic resonance method (ASR) for the sun gear fault detection. Smidt and Ryan [10] mounted an internal sensor on the planet carrier for data collection and applied the TSA technique to the internal vibration signal for the fault detection of planetary gears. The results showed that the most obvious advantage of internal sensor based measurement was that the raw signal can be directly converted to the useful damage indicator for gear faults, such as kurtosis or root-mean-square (RMS) values, without any preprocessing steps.

While many studies on monitoring gear faults have been published, it seems that there is still lack of effective approaches for the fault diagnosis of planetary bearings. This 
is because the bearing related vibration signals are strongly masked by gear signals. In addition, the signal from planet gear bearings is transmitted through the ring gear to the fixed external sensor via a time varying transmission path, which means that the fault signal is subject to both noise contamination and modulation [2]. In order to overcome these problems, Smith et al. [11] employed an internal accelerometer mounted on the planet carrier and an externally fixed accelerometer for identifying planetary gear bearing defects. It was demonstrated that the internal sensor based measurement has potential superiority in inner race fault detection over the external sensor, while both the external sensor measurement and internal sensor measurement provided similar diagnostic performance for the outer race fault. It was also pointed out in [10] that a limitation of internal sensor based measurement was that the signal might be contaminated by electromagnetic interference due to the use of a slip ring for the signal transmission.

This paper presents further development of fault diagnosis approaches by using the internal vibration sensor. It aims to improve the fault detection effectiveness by solving the problems of demodulation band selection of internal sensor signals caused by electromagnetic interference. The new fault diagnosis method, which is developed by employing several advanced digital signal processing (DSP) techniques, will be introduced. The performance of the proposed approach on detecting both the inner race faults and outer race faults of planetary bearings will be assessed. 


\section{Methodology}

\subsection{Sensing by using internal vibration sensor}

For vibration-based condition monitoring of planetary bearings, the traditional mounting location is on the external housing or the ring gear for the ease of installation and maintenance. However, bearing fault signals from the external sensor measurement are attenuated after the transmission through the gear mesh between the planet gear and ring gear. Moreover, the transmission path from the source of fault location to the externally mounted stationary accelerometer is time-varying. This varying path will lead to the modulation of the key vibration signals.

A new approach is to mount an accelerometer internally on the planet carrier to solve the issues of variable transmission path and receiving more direct vibration signals. A slip ring is used for the transmission of the vibration signal from the internally mounted sensor. A schematic view of the external and internal sensor locations is illustrated in Figure 1.

However, due to the use of a slip ring for signal transmission, the internal accelerometer signals are contaminated by electromagnetic interference in the high frequency range, as shown in Figure 2. The interference may come from the variable frequency controller and/or the ground loop effect. Therefore, as illustrated in Figure 3, the bearing fault signal is masked by deterministic gear signals, random noise and the electromagnetic interference. 


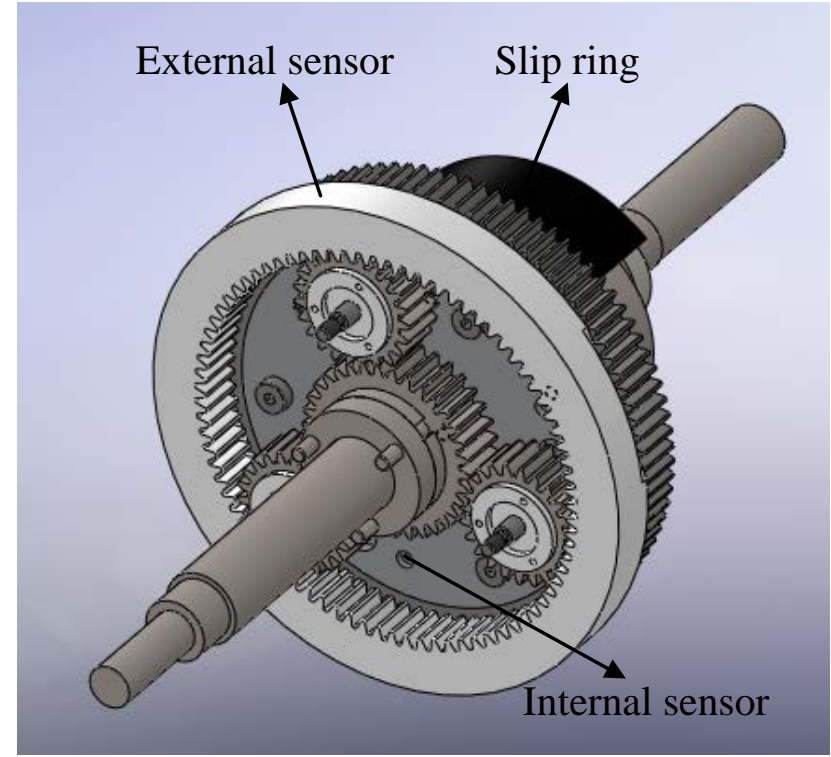

Figure 1 A schematic for the locations of accelerometers

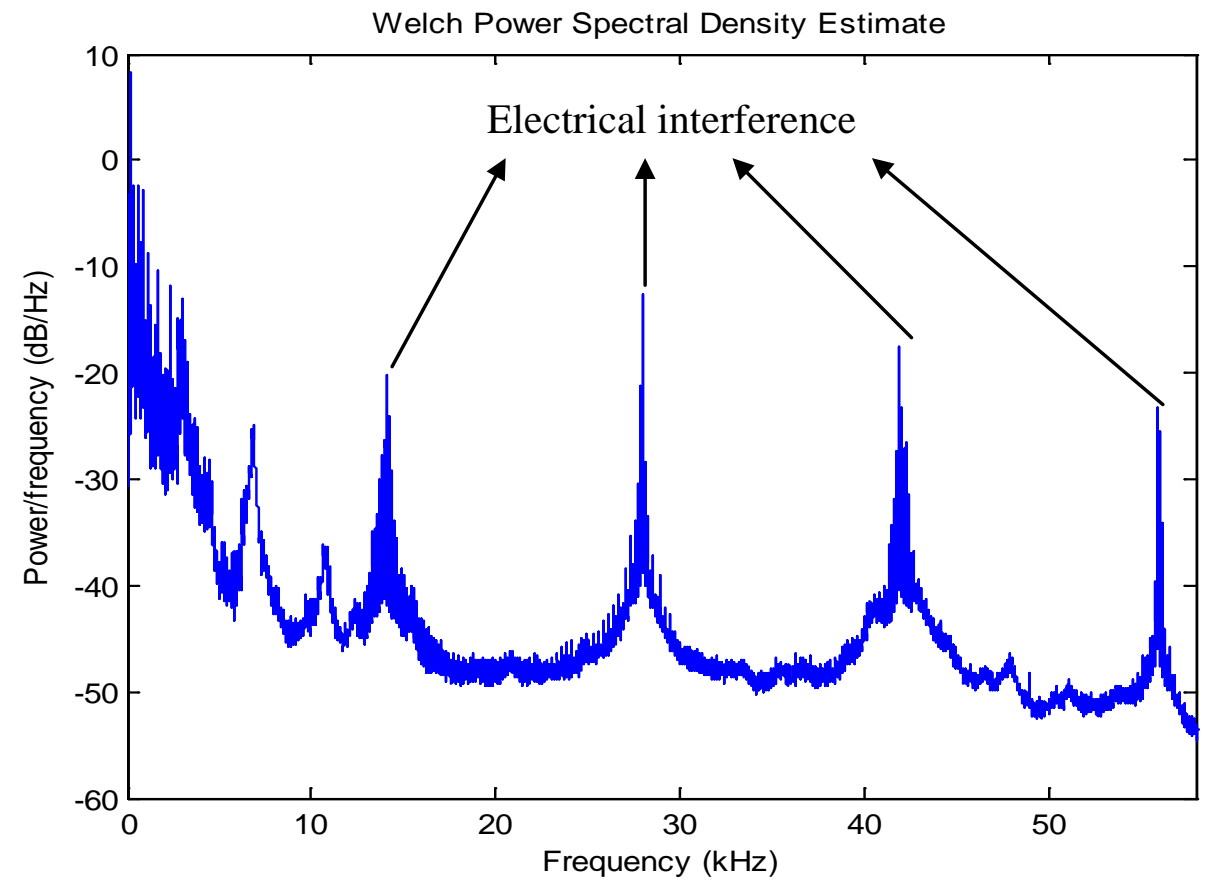

Figure 2 Power spectral density of internal acceleration signal 


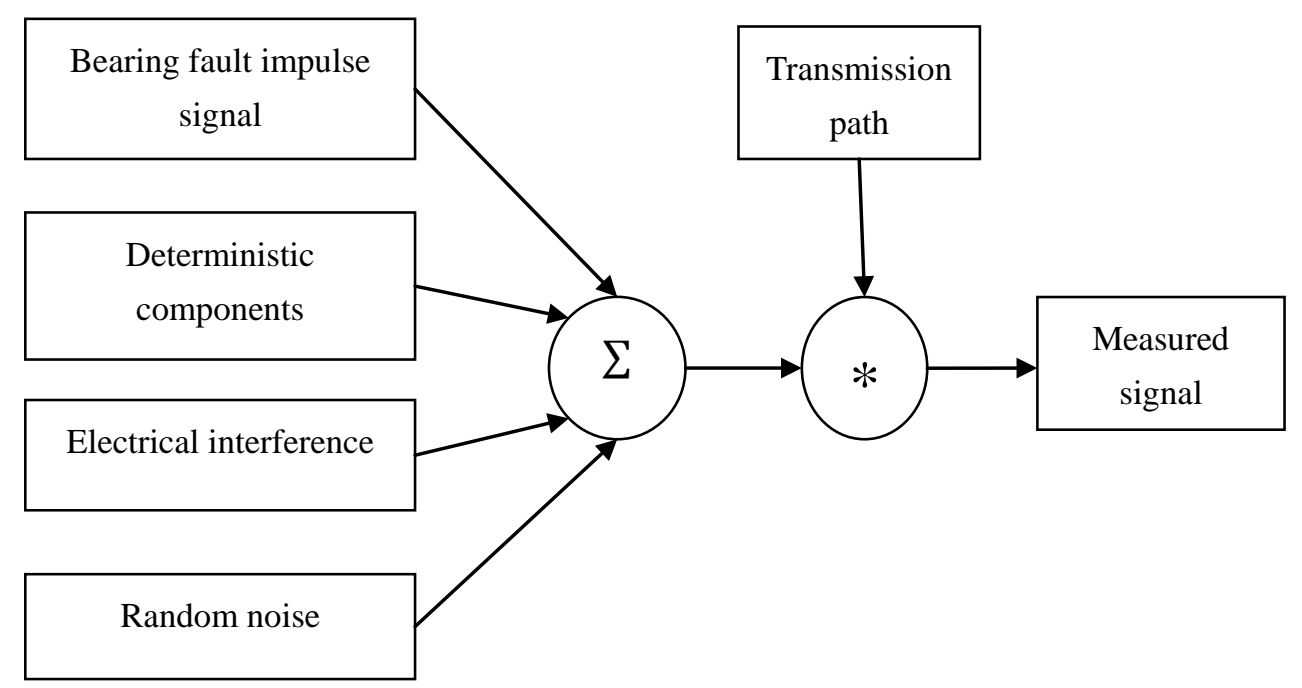

Figure 3 A schematic for the signal and noise detected by the internal accelerometer

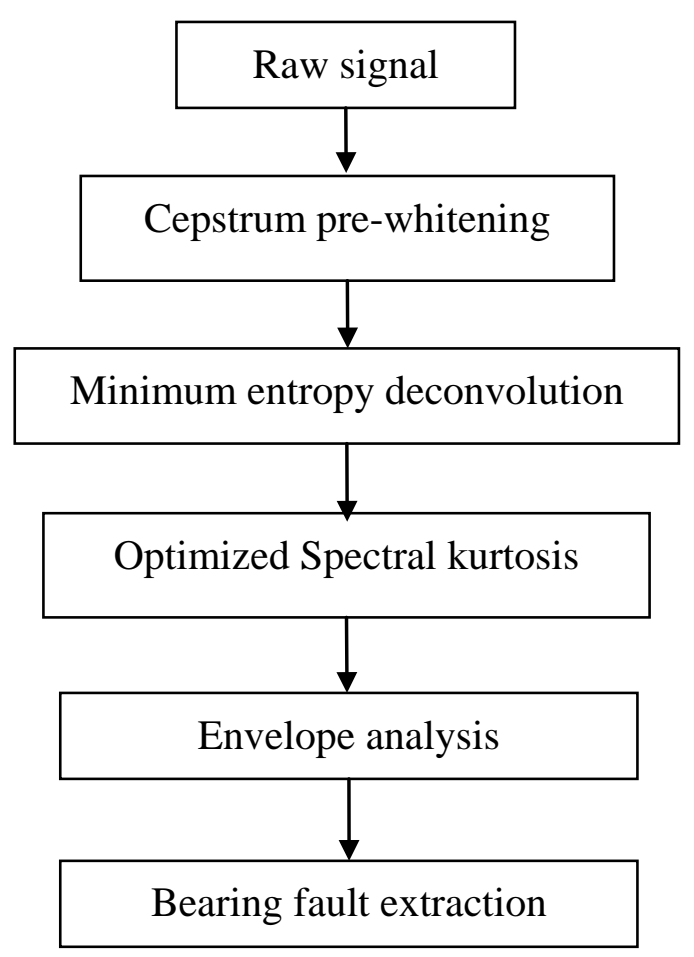

Figure 4 Flow chart of diagnostic procedure

To filter out undesired components in the measured signal and to improve the signal to noise ratio (SNR), a hybrid signal processing approach is developed as shown in Figure 4. Cepstrum pre-whitening is the first step for removing the discrete 
frequencies from the raw vibration signal. Minimum entropy deconvolution (MED) is then applied to remove the effect of transmission path and to enhance the bearing fault related impulsiveness. Since the Fast Kurtogram based spectral kurtosis (SK) is easily affected by the large SK value associated with the electromagnetic interference, the selection of the demodulation band may be misled by the results of the Fast Kurtogram. An optimized spectral kurtosis method is utilized as the third step for the selection of the demodulation band. Envelope analysis is the final step which is used for the extraction of bearing fault information in the squared envelope spectrum.

\subsection{Cepstrum pre-whitening (CPW)}

Cepstrum pre-whitening (CPW) is a method for separating discrete components and random components from a signal [12]. The main interest in the signals from a faulty bearing is the random part rather than the deterministic part, thus it is a wise choice to filter out all deterministic components to facilitate bearing diagnosis. Comparing to the traditional separation techniques such as time synchronous averaging (TSA) [13], self-adaptive noise cancellation (SANC) [14], and discrete random separation (DRS) [4], CPW is much simpler and tracking is not required. Moreover, it can remove both harmonics and modulation sidebands [12]. CPW is based on the real cepstrum [15]. The Cepstrum $C[n]$ is calculated as the inverse discrete Fourier transform (IDFT) of the log magnitude of the DFT of a signal $x[n]$, which can be expressed as:

$$
C[n]=I D F T\{\log |\operatorname{DFT}(x[n])|\}
$$


Based on the above expression, the periodicity in the signal can produce peaks at the quefrency in the real cepstrum domain which means that peaks in the absolute values of the real cepstrum can predict the presence of the deterministic components in the raw signal. In view of this inference, the CPW technique sets all values to zero in the real cepstrum domain to give all deterministic components in the frequency domain the same amplitude, meaning that the discrete frequency components no longer protrude from noise values and are dominated by the latter. On the other hand, for signals from a faulty bearing, there exist components related to the bearing fault, which are cyclostationary rather than periodic. The cyclostationary components are left after the liftering process in the CPW and can be used for bearing fault detection through further analysis. The process of CPW is shown in Figure 5.

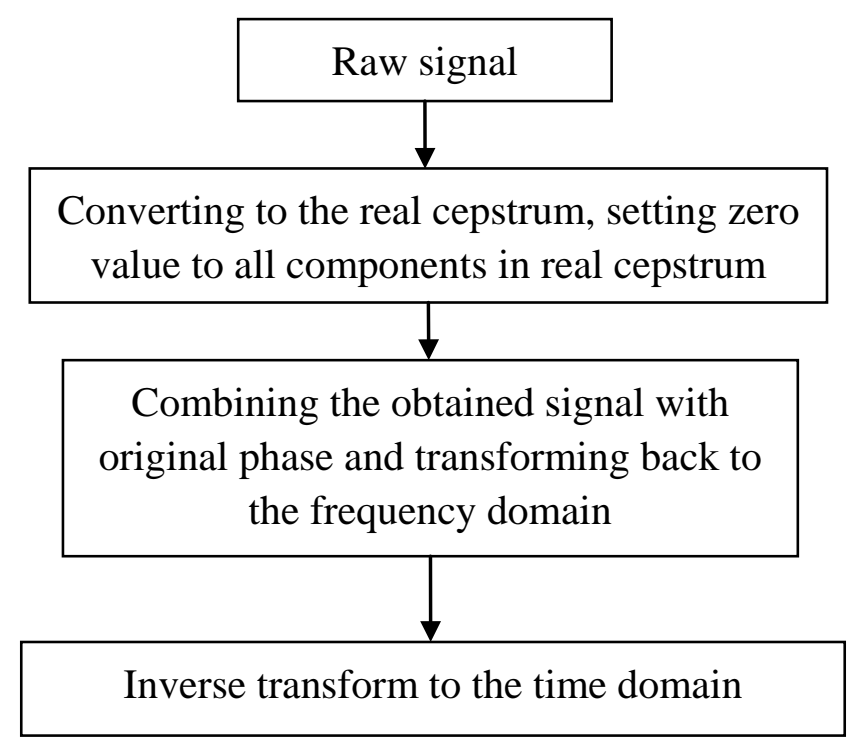

Figure 5 Procedure of Cepstrum pre-whitening

\subsection{Minimum entropy deconvolution (MED)}

The MED technique was initially developed as an auxiliary tool for extracting 
reflectivity information from seismic data [16]. Due to its validity in deconvolving impulsive excitations from the response signals, MED was applied by Endo and Randall [17] for enhancing the impulses generated from the local defects of gears. It was further applied to optimizing bearing fault detection by increasing impulses caused by localized bearing faults [18].

The main idea of the MED technique is to produce an inverse filter for neutralizing the effect of the signal transmission path and searching for the best set of filter coefficients that can recover the signal with the minimum value of entropy [17]. As explained in [18], a signal becomes more disordered and less structured when the entropy value is higher. Hence, minimizing the value of entropy has the effect in improving the structured information from the original signal and thus enhancing the impulsiveness (kurtosis) for the MED filter output signal. The process of forming a MED filter [19] is summarized as follows.

Generating a FIR filter $f$ with $L$ coefficients that make:

$$
y(\mathrm{n})=\sum_{l=1}^{L} f(l) v(n-l)
$$

Where $L$ is the length of the deconvolution filter $f, v$ is the input signal and $y$ is the output signal, the deconvolution filter $f$ is able to inverse the structural filter $h$ that achieves:

$$
f * h(n)=\delta\left(n-l_{m}\right)
$$

The inverse filter $f$ can be causal with the delay $l_{m}$. 
In order to obtain the output signal with the minimum entropy, the objective function method (OFM) is adopted in the MED filter as an optimizing process for maximizing the kurtosis of the signal after MED filtering $[17,18]$. The process is as below.

(1) The kurtosis of the output signal $y(n)$ is selected as the objective function $O_{k}(f)$ to be maximized:

$$
O_{k}(f)=\left[\sum_{n=0}^{N-1} y^{4}(n)\right] /\left[\sum_{n=0}^{N-1} y^{2}(n)\right]^{2}
$$

(2) Determining the filter coefficients of the MED filter $f$ by setting the derivative of the objective function as zero, where an iteration process is needed:

$$
\partial O_{k}(f) / \partial f=0
$$

(3) The iteration stops when the expected value of error is less than the set value of tolerance. The optimizing filter coefficient vector $f$ for MED filter is then selected.

\subsection{Optimized Spectral Kurtosis}

The spectral kurtosis (SK) was firstly proposed by Antoni and Randall [20] into the condition monitoring of rotating machines as a defect indicator for detecting the incipient faults which generate impulse-like signals, and indicating their locations in the frequency domain. SK is estimated based on the short-time Fourier transform (STFT) that can be expressed as [21]:

$$
K(f)=\left\langle|X(t, f)|^{4}\right\rangle /\left\langle|X(t, f)|^{2}\right\rangle^{2}-2
$$


Where $X(t, f)$ is the time/frequency envelope of the original signal and the square of its amplitude means the power spectrum values at each time. $\langle$.$\rangle is the$ time-averaging operator.

Due to the reason that the SK value varies with both the frequency $f$ and frequency resolution $\Delta f$, a representation of SK in the $(f, \Delta f)$ plane is required for finding the maximum value of SK. However, the full exploration of each SK value from the $(f, \Delta f)$ plane is infeasible as it is time-consuming and thus hard to be applied to industrial applications. To solve the issues mentioned above, the Fast Kurtogram was introduced in [22] as a further development of the SK method for fast computing the SK values over dyadic grid in the $(f, \Delta f)$ plane.

Although the Fast Kurtogram has been proved to give successful diagnostic results in many cases [19, 22], it may lead to a wrong choice of the demodulation band when some other extraneous vibration sources or noise dominate the impulsiveness of the vibration signals $[15,23]$. When using internal sensor based measurement, it has been shown [11] that the vibration signal from the internal sensor was seriously contaminated by the electromagnetic interference from the variable frequency drive (VFD), and that applying the Fast Kurtogram could generate misleading results. An optimized spectral kurtosis is proposed in this study to solve this problem. Inspired by an approach in [23], the optimized spectral kurtosis method still uses the spectral kurtosis as the defect indicator for bearing fault diagnosis. Instead of creating a 
$(f, \Delta f)$ plane in the Fast Kurtogram, this method firstly sets the bandwidth value (frequency resolution) covering 1-5 harmonic lines of the characteristic fault frequencies of the defect elements, and then chooses the optimum center frequency for demodulation.

\subsection{Envelope analysis}

Envelope analysis is one of the most practical methods in bearing diagnosis. The characteristic defect frequencies are easier to be recognized in the envelope spectrum than in the frequency spectrum, because the wide distribution energy of the impulse has very small values at the fault repetition frequencies. On the other hand, the fault impulses are amplified by structural resonances in higher frequency bands. Therefore, analyzing the envelope signal is more effective in obtaining the desired diagnostic information [24].

The Hilbert transform has been used to carry out the amplitude demodulation in the envelope analysis [19]. By applying a Hilbert transform to the vibration signal $\mathrm{x}(\mathrm{t})$ to calculate its analytic form, the imaginary part of the analytic signal is obtained as:

$$
H[x(t)]=\frac{1}{\pi} \int_{-\infty}^{\infty} x(\tau) /(t-\tau) d \tau
$$

The analytical signal z(t) can be constructed as:

$$
z(\mathrm{t})=\mathrm{x}(\mathrm{t})+j H[x(\mathrm{t})]
$$


Then $e(t)$, which is the envelope of $z(t)$, is calculated as:

$$
e(\mathrm{t})=\sqrt{x^{2}(t)+H^{2}[x(\mathrm{t})]}
$$

The performance of the envelope analysis is further improved by squaring the envelope signal. This is because that the squaring process has the benefit of enhancing the bearing frequency components in envelope spectrum and degrading the background noise [25].

\section{Experimental Setup}

The planetary gearbox test rig used in this study is shown in Figure 6. The speed of the driving shaft was controlled by a 3 Phase AC motor with a soft-start frequency controller. The frequency controller also allowed the speed of the rig to be accurately modulated during operation. A hydraulic system was used to provide a resistance torque to the gearbox. A torque transducer was employed to measure the applied torque.

A schematic of the planetary gearbox is shown in Figure 7. It consisted of one-stage parallel shaft and one-stage planetary. The parallel shaft stage included a pinion gear of 32 teeth meshing with a large spur gear of 90 teeth, which was connected directly to the planet carrier. The planetary stage included an 80 tooth ring gear, three 20 tooth planetary gears, and a 40 tooth sun gear to give the planetary stage a gear ratio of 1:3. The ring gear is fixed while the three planet gears mesh with the ring gear and the sun gear simultaneously. An overall gear ratio of the gearbox was 1:1.0667. Grease lubrication 
was used in the gearbox to allow the slip ring to be placed inside the housing.

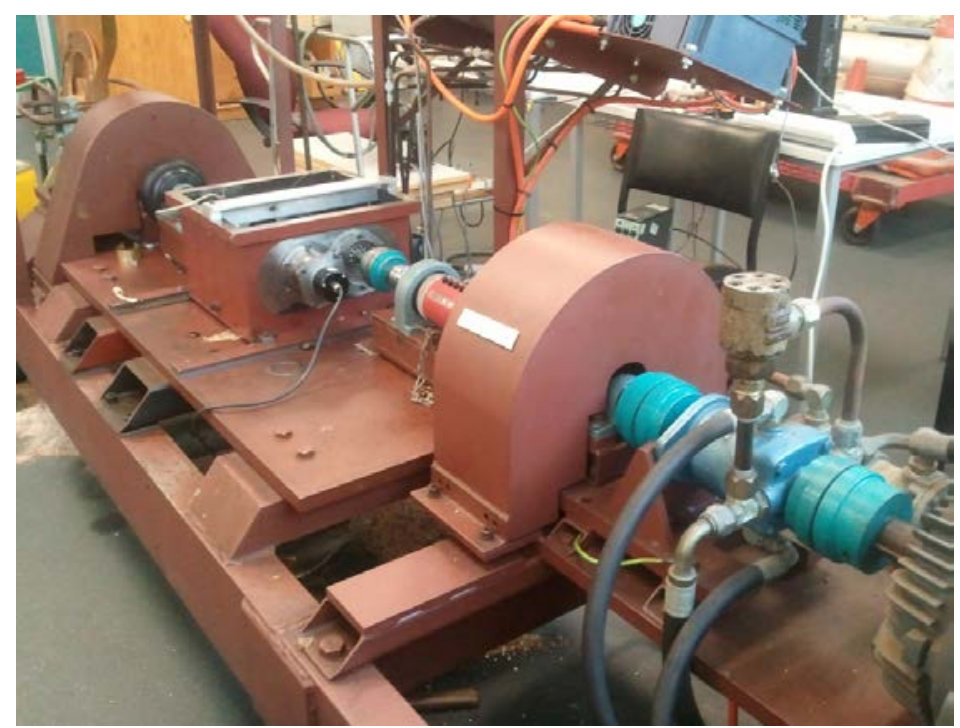

Figure 6 Planetary gearbox test rig

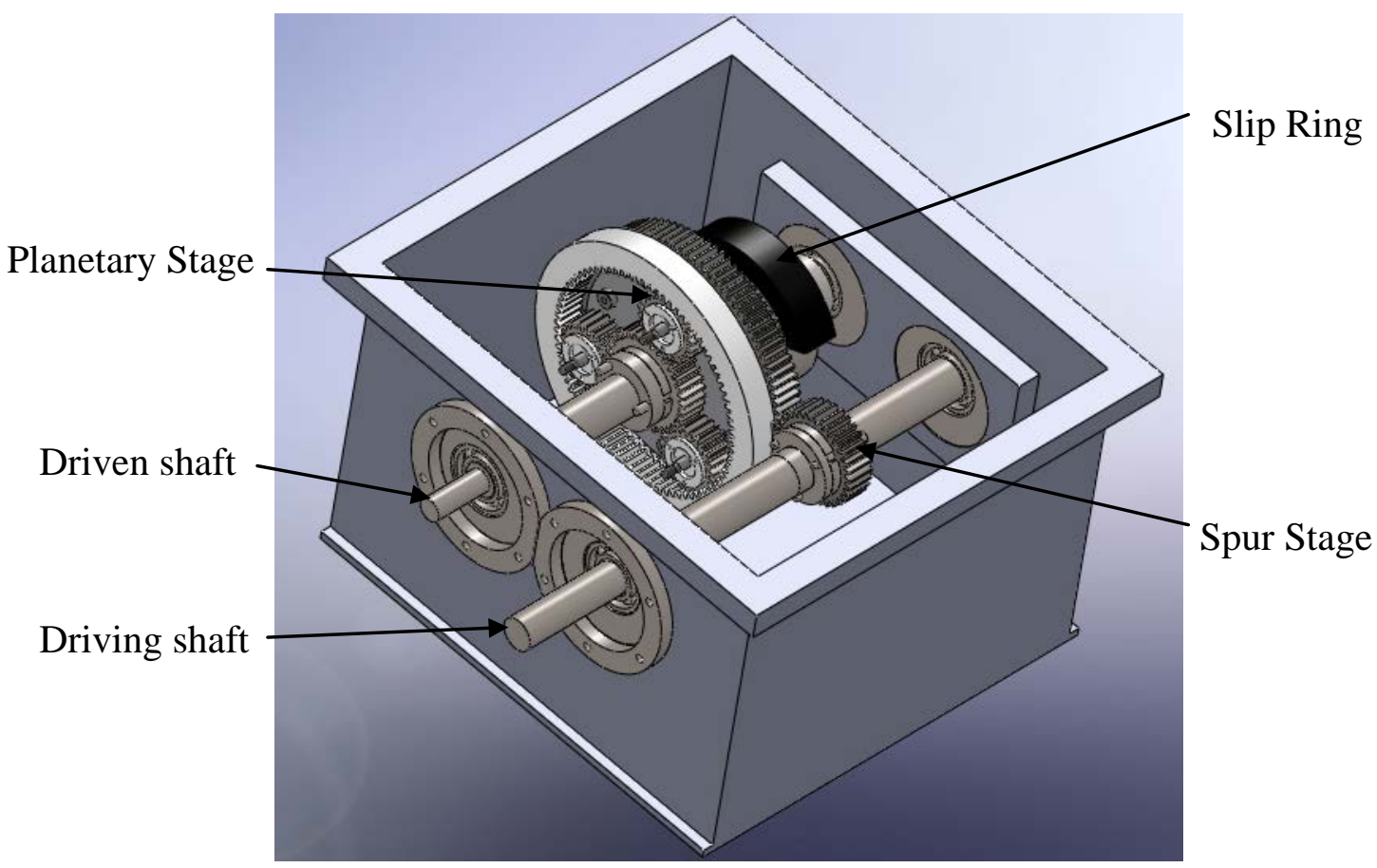

Figure 7 CAD model of the planetary gearbox

Two sets of B\&K 4394 IEPE-type accelerometers were used in both the internal and external vibration measurements. The external accelerometer was mounted on the outer surface of the ring gear in the radial direction, and the internal accelerometer 
was mounted on the planet carrier in the axial direction. Since the internal accelerometer rotated with the planet carrier, a Michigan Scientific B6-2 slip ring was used for transmitting the vibration signal from the internal accelerometer to the signal conditioner. A B\&K PULSE front end was connected with a laptop running B\&K Labshop for data capturing and recording. The recorded data were exported to MATLAB for further signal processing.

IKO NAF 122413 needle roller bearings were used as the planet gear bearings. Each contained 15 roller pins of $2 \mathrm{~mm}$ diameter, and the bearing pitch diameter was $18 \mathrm{~mm}$. Both the inner race fault and outer race fault were seeded in the planet gear bearings, which were used respectively in the testing. The technique of electric spark erosion was used for the fault seeding. The notch width of the outer race was $1.2 \mathrm{~mm}$ with a depth of $0.4 \mathrm{~mm}$, while the notch on the inner races was $1.0 \mathrm{~mm}$ width with the depth of $0.4 \mathrm{~mm}$.

During the operation of the gearbox rig, testing was conducted at a constant input shaft speed of $6 \mathrm{~Hz}$ with different torque loads including 30, 50 and 70Nm. Also, testing with bearings in different status (good bearing, inner race fault bearing, outer race fault bearing) was conducted. In each test, the vibration signals were collected for both the internal accelerometer and external accelerometer. A matrix of the test conditions is shown in Table 1. 
Table 1 Test conditions of the gearbox rig

\begin{tabular}{cccc}
\hline Test number & Input shaft Speed $(\mathrm{Hz})$ & Load $(\mathrm{Nm})$ & Bearing status \\
\hline 1 & 6 & 30 & Good \\
\hline 2 & 6 & 30 & Inner race fault \\
\hline 3 & 6 & 30 & Outer race fault \\
\hline 4 & 6 & 50 & Good \\
\hline 5 & 6 & 50 & Inner race fault \\
\hline 6 & 6 & 50 & Outer race fault \\
\hline 7 & 6 & 70 & Good \\
\hline 8 & 6 & 70 & Inner race fault \\
\hline 9 & 6 & 70 & Outer race fault \\
\hline
\end{tabular}

\section{Results and discussion}

\subsection{Bearing fault frequencies}

For a given rolling element bearing with a defect, a series of impacts are generated when each rolling element passes through the defect location. Unlike a conventional bearing configuration where the outer race is stationary and the inner race rotates at the shaft speed, for a planetary bearing, the outer race rotates at the speed of the planet gear while the inner race rotates in the opposite direction at the speed of the planet carrier (but with a fixed orientation with respect to the load). Given the basic geometry of the planet bearing (Table 2), the equations for calculating the bearing characteristic defect frequencies are shown below:

Table 2 Planet gear bearing parameters

\begin{tabular}{ll}
\hline Type & IKO NAF 122413 \\
\hline Ball diameter $(d)$ & $2 \mathrm{~mm}$ \\
\hline Pitch circle diameter $(D)$ & $18 \mathrm{~mm}$ \\
\hline Number of rolling elements $(n)$ & 15 \\
\hline Contact angle $(\beta)$ & $0^{\circ}$ \\
\hline
\end{tabular}


Ball pass frequency, outer race $(B P F O)$ :

$$
B P F O=\frac{n}{2}\left\{f_{i}\left(1-\frac{d}{D} \cos \beta\right)-f_{o}\left(1+\frac{d}{D} \cos \beta\right)\right\}
$$

Ball pass frequency, inner race $(B P F I)$ :

$$
B P F I=\frac{n}{2}\left\{f_{i}\left(1+\frac{d}{D} \cos \beta\right)-f_{o}\left(1+\frac{d}{D} \cos \beta\right)\right\}
$$

Where $n$ is the number of rolling elements, $\beta$ is the angle of the load from the radial plane, $d$ is the diameter of the roller, $D$ is the pitch diameter of the bearing, $f_{o}$ is the planetary bearing outer race speed, and $f_{i}$ is the inner race speed. In this case with the input shaft speed of $6 \mathrm{~Hz}$, it can be found that $f_{o}=8.52 \mathrm{~Hz}$, and $f_{i}=2.13 \mathrm{~Hz}$. The resultant defect frequencies are $B P F O=56.87 \mathrm{~Hz}$, and $B P F I=70.93 \mathrm{~Hz}$. The inner race fault signal is unmodulated (by rotation of the gear), while the outer race fault signal is modulated by the relative frequency of the inner and outer races as it passes through the load zone.

\subsection{Diagnosis of outer race fault}

The characteristic frequency for the outer race fault was $56.87 \mathrm{~Hz}$ when the rotating speed of the input shaft was $360 \mathrm{RPM}$. In the case of $70 \mathrm{Nm}$ torque load, the proposed signal processing approach was applied to the vibration signal acquired from the internal accelerometer. As shown in Figure 8, the impulsiveness became much clearer after the Cepstrum whitening and MED filtering. The next step was to use the SK method to look for the best demodulation band. An optimal bandwidth of $512 \mathrm{~Hz}$ was 
used on the basis of the band selection criteria presented in [23]. The optimized SK is shown in Figure 9, where the optimal center frequency for demodulation was 44360 $\mathrm{Hz}$ identified by its maximum kurtosis value. Envelope analysis was then conducted at the selected high-frequency band for demodulation and fault information pick-up. The outer race fault frequency $55.1 \mathrm{~Hz}$ (a slight difference due to actual slip in the bearing) of the planet bearing can be clearly identified in the squared envelope spectrum shown in Figure 10. The same band of the good bearing is also presented in Figure 11 for comparison (note difference in amplitude scale). It is noted that the 100 $\mathrm{Hz}$ components, $24 \mathrm{~Hz}$ components and their harmonics in this figure are believed to be caused by the electromagnetic interference.

(a) kurtosis $=3.5945$

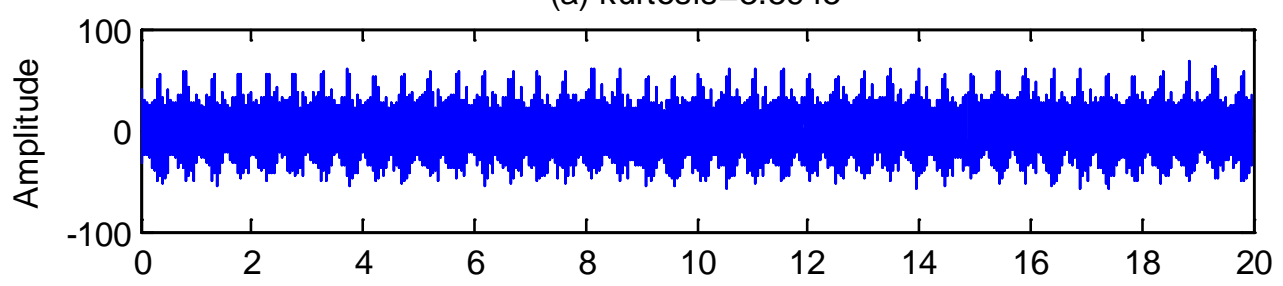

(b) kurtosis $=7.7933$

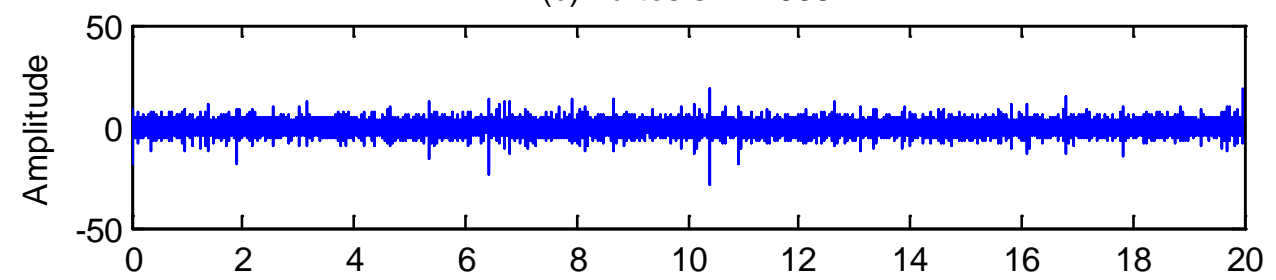

(c) kurtosis $=43.0941$

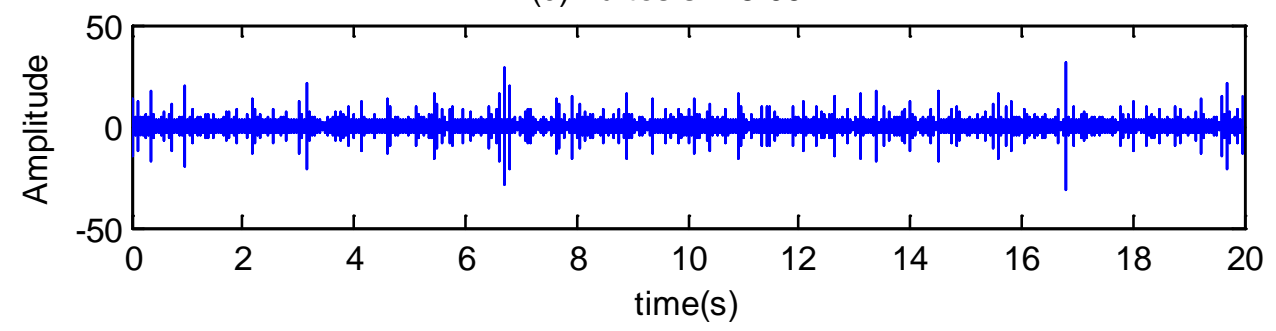

Figure 8 (a) raw time signal; (b) Cepstrum whitened signal; (c) signal after Cepstrum whitening and MED filtering 


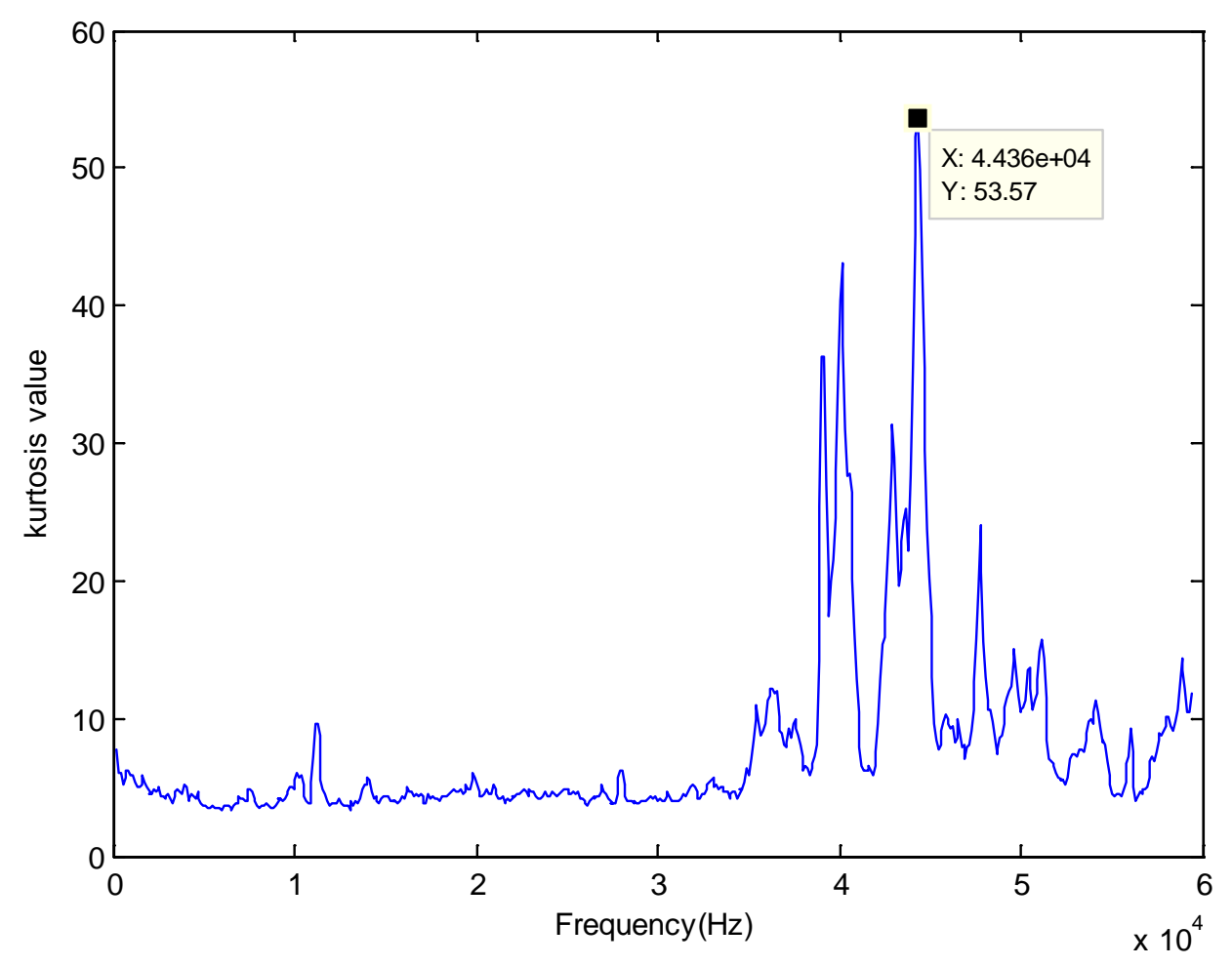

Figure 9 Optimized SK

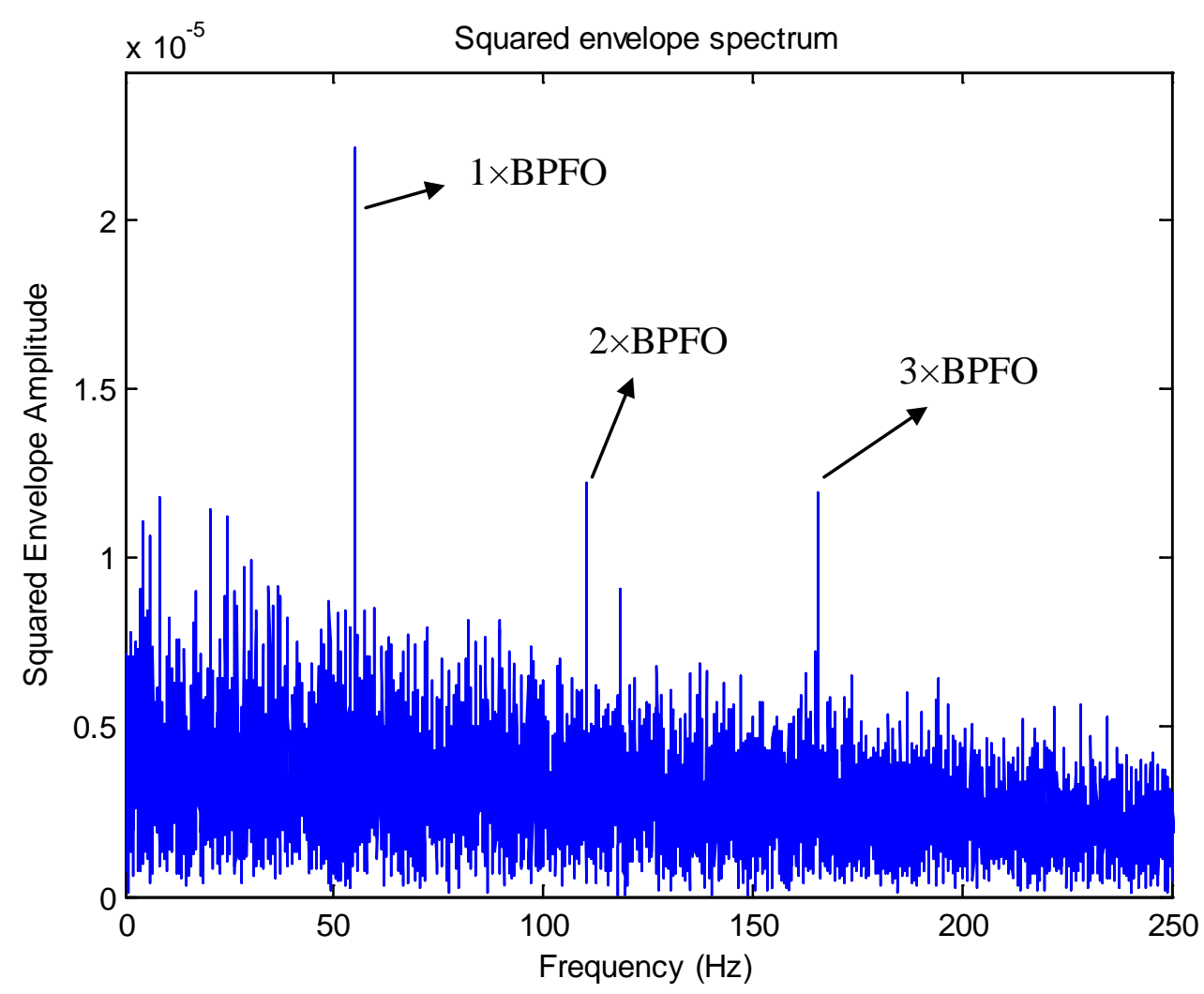

Figure 10 Squared envelope spectrum 


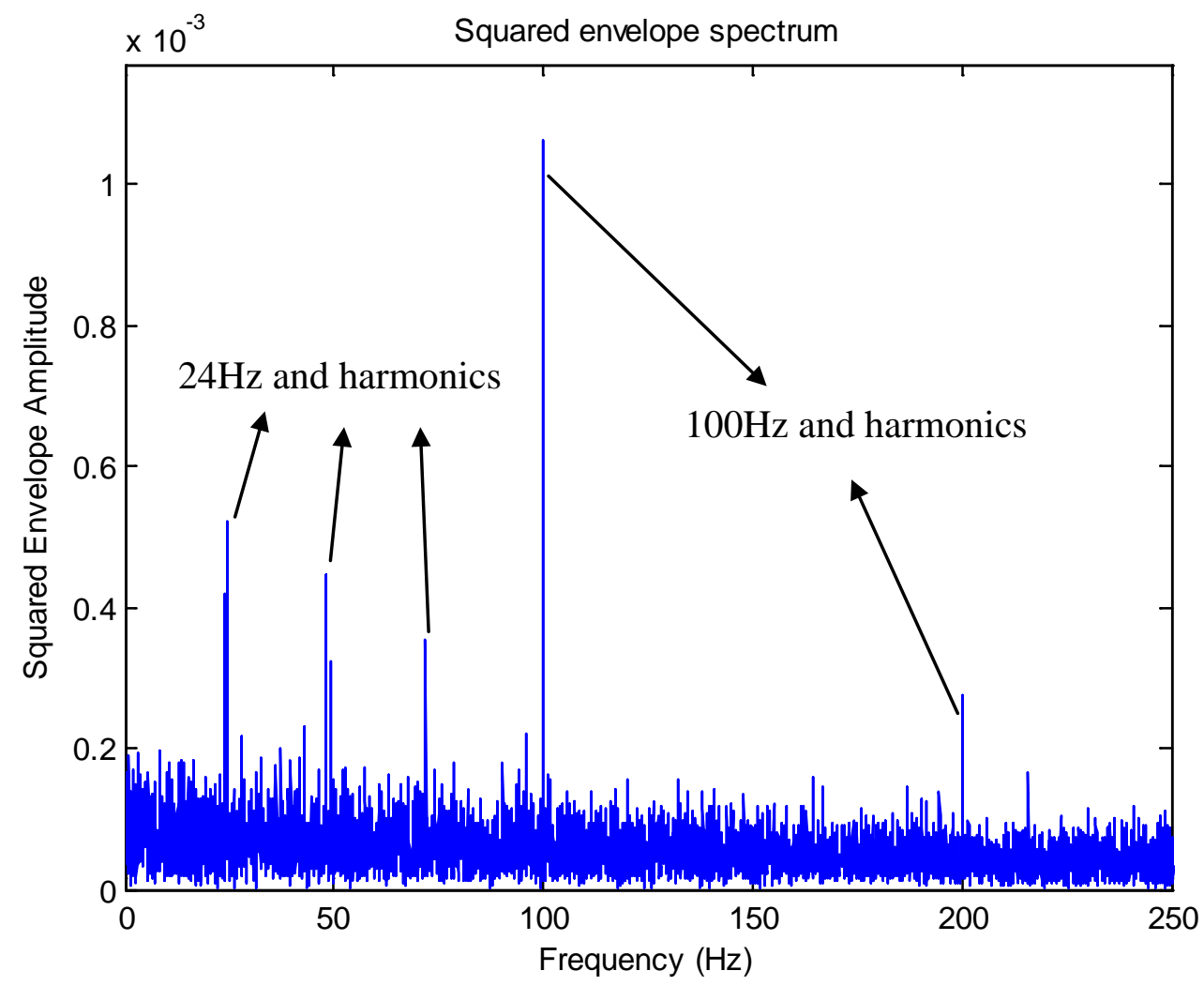

Figure 11 Squared envelope spectrum (Good bearing)

The optimized SK and the squared envelope spectrum of the external sensor based measurement are presented in Figure 12 and Figure 13 respectively. The results show that the external sensor based measurement also can recognize the outer race fault in planetary bearings, although the squared envelope spectrum from the external sensor signal is much noisier. A summary of the diagnostic performance for outer race fault using both the external sensor and internal sensor is given in Table 3. It is noted that while both sensor configurations can successfully detect the outer race fault, the demodulation bands are different for the internal and external sensors. 


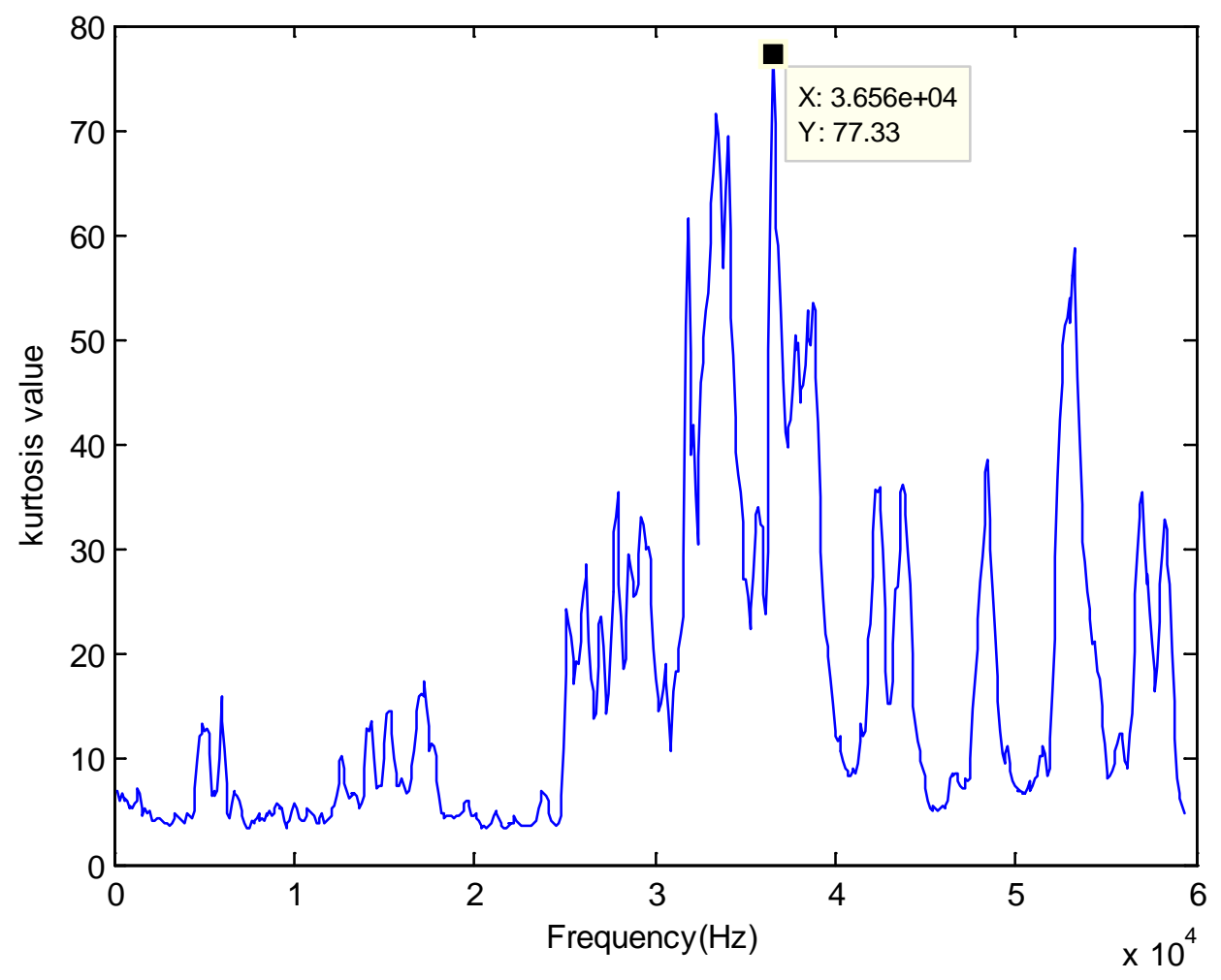

Figure 12 Optimized SK (External sensor)

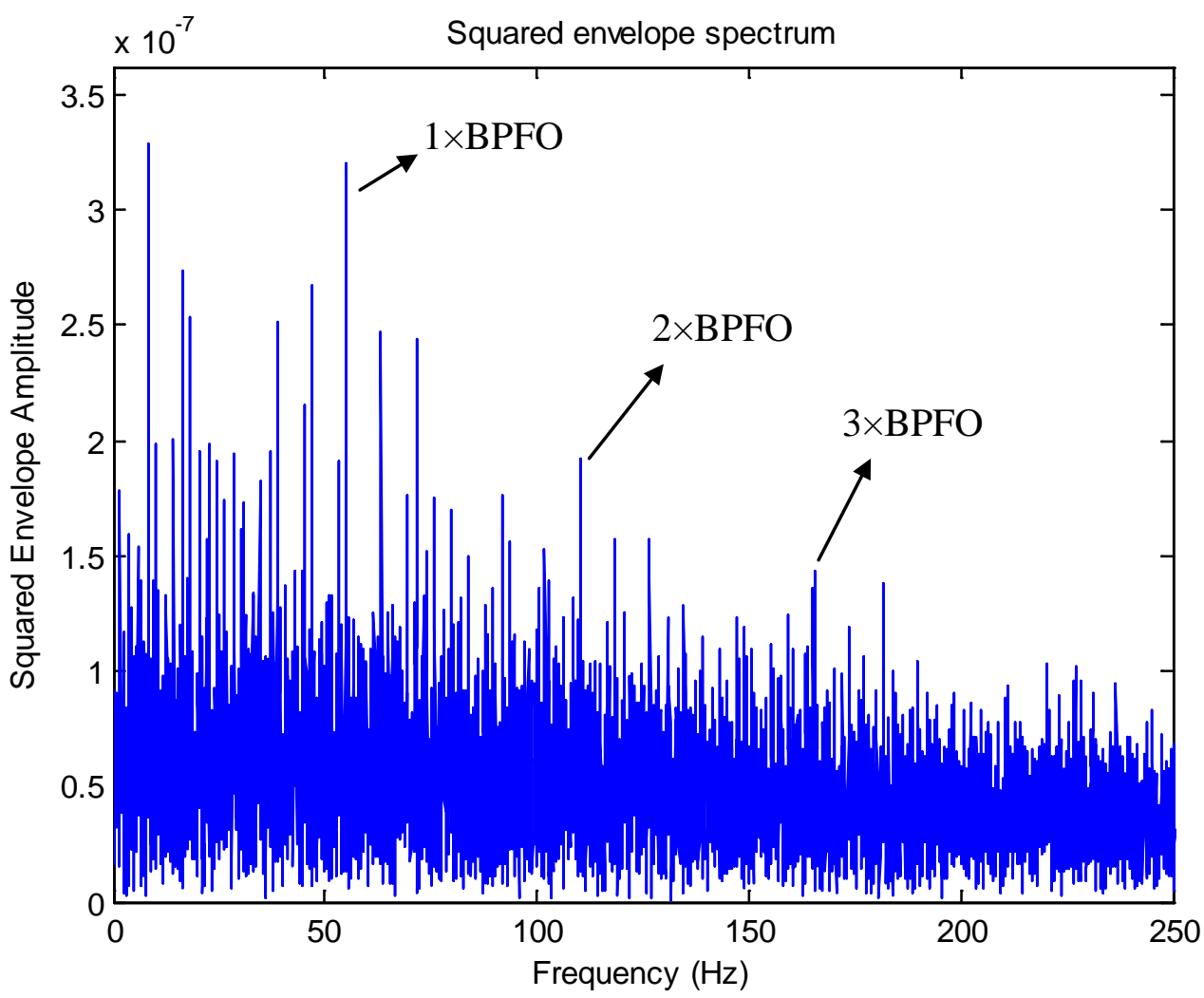

Figure 13 Squared envelope spectrum (External sensor) 
Table 3 Diagnostic results for tests with outer race faults

\begin{tabular}{cccc}
\hline Torque load(Nm) & Sensor location & $\begin{array}{c}\text { Demodulation band } \\
(\mathrm{Hz})\end{array}$ & $\begin{array}{c}\text { Diagnosis } \\
\text { performance }\end{array}$ \\
\hline \multirow{2}{*}{30} & External & $38354-38866$ & success \\
\cline { 2 - 4 } & internal & $43904-44416$ & success \\
\hline \multirow{2}{*}{50} & External & $33754-34266$ & success \\
\cline { 2 - 4 } & internal & $40104-40616$ & success \\
\hline 70 & External & $36304-36816$ & success \\
\cline { 2 - 4 } & internal & $44104-44616$ & success \\
\hline
\end{tabular}

\subsection{Inner race fault diagnosis}

The same signal processing procedures were applied to the inner race fault signal in the case of $70 \mathrm{Nm}$ torque load, in which the characteristic frequency was $70.93 \mathrm{~Hz}$ when the input shaft speed was 360 RPM. For internal sensor based measurement, the impulsiveness was greatly increased after Cepstrum whitening and MED filtering (Figure 14). In the next step of optimized SK as shown in Figure 15, the optimal center frequency for demodulation was $46960 \mathrm{~Hz}$ due to its maximum kurtosis value. Envelope analysis was then applied at the selected high-frequency band for demodulation and fault information extraction. In Figure 16, the inner race fault frequency $68.15 \mathrm{~Hz}$ (a slight difference due to actual slip in the bearing) of the planet bearing can be clearly recognized in the squared envelope spectrum with the corresponding peak value. The squared envelope spectrum of the good bearing in the same band is presented in Figure $\mathbf{1 7}$ as a comparison. No peaks at the BPFI and harmonics can be identified. Due to the contamination by the electromagnetic interference, the $100 \mathrm{~Hz}$ components, $24 \mathrm{~Hz}$ components and their harmonics were dominant in this figure. 
(a) kurtosis $=3.5747$

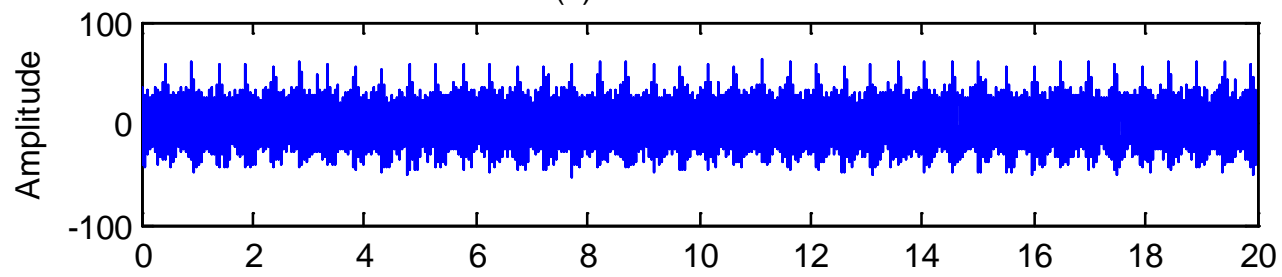

(b) kurtosis $=13.7814$

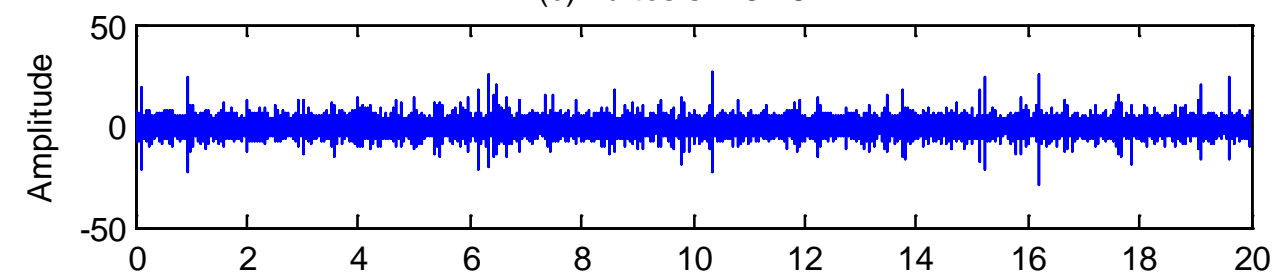

(c) kurtosis $=137.6622$

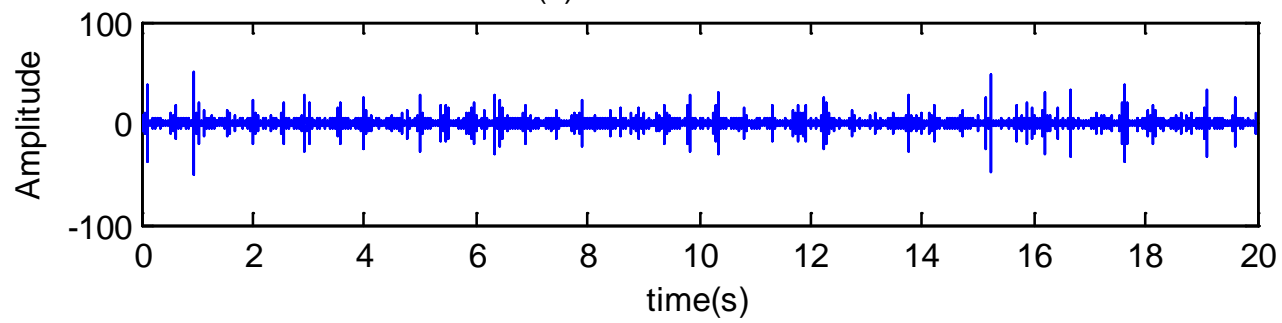

Figure 14 (a) raw time signal from internal sensor; (b) Cepstrum whitened signal; (c) signal after Cepstrum whitening and MED filtering

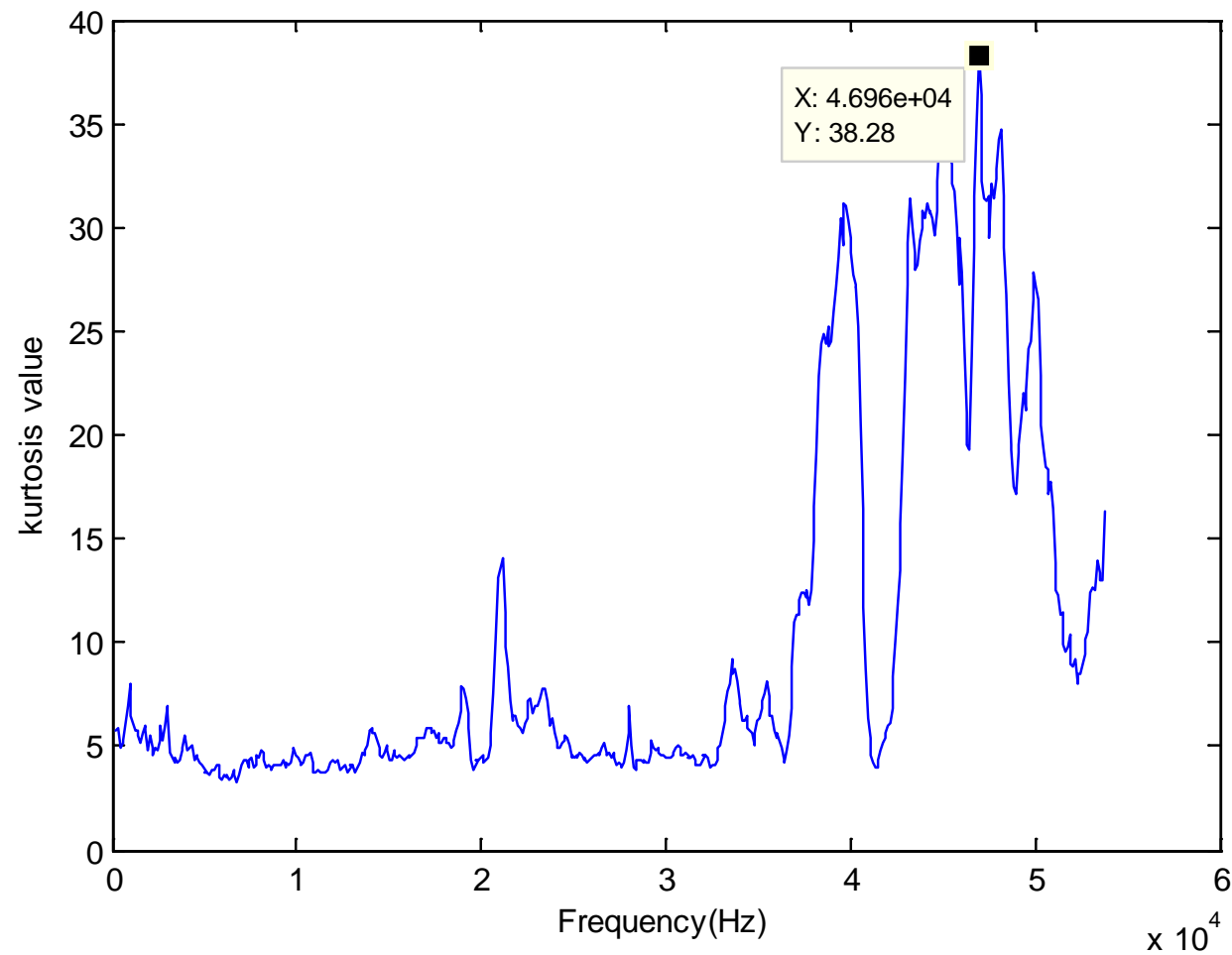

Figure 15 Optimized spectral kurtosis (SK) (internal sensor) 


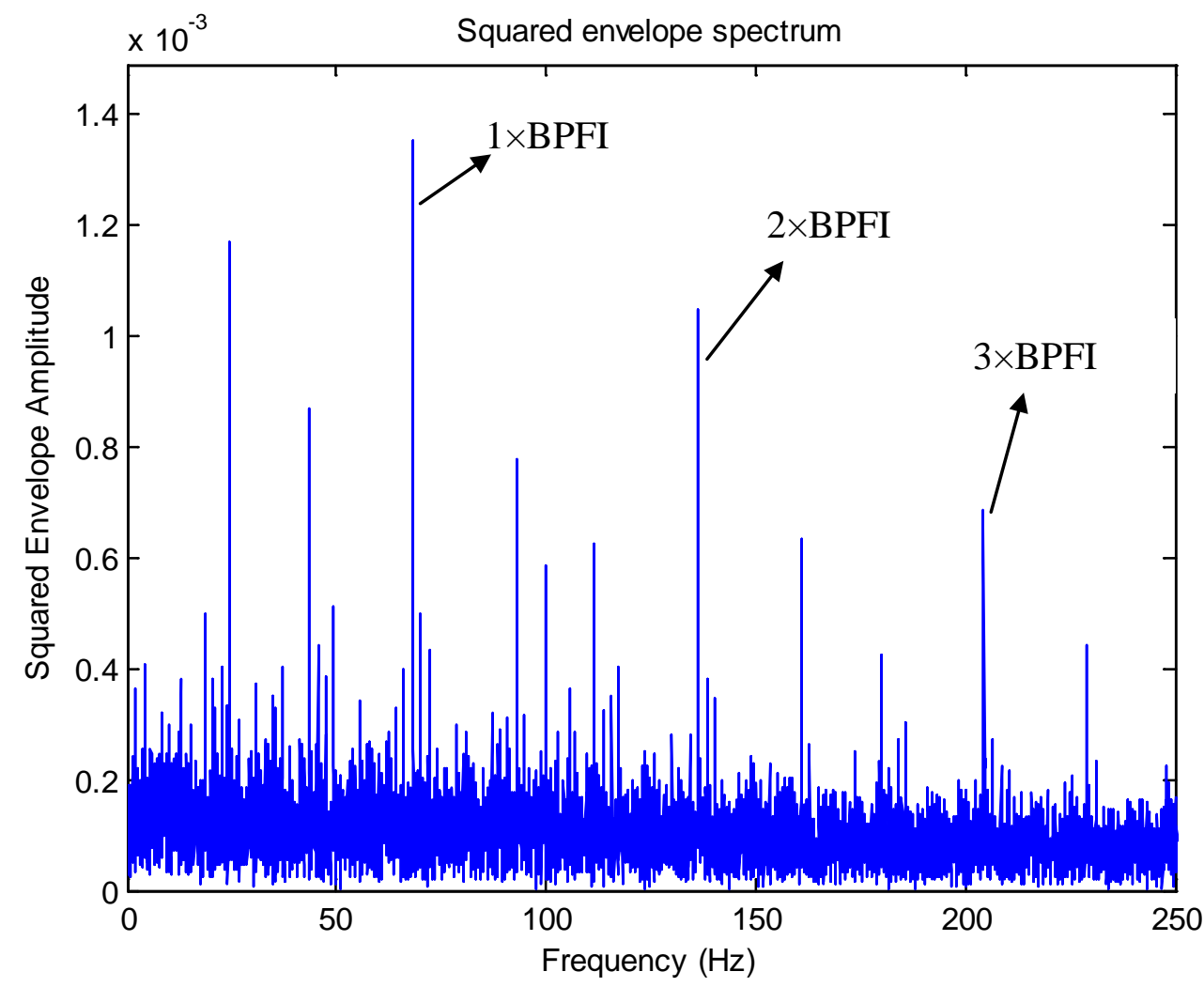

Figure 16 Squared envelope spectrum

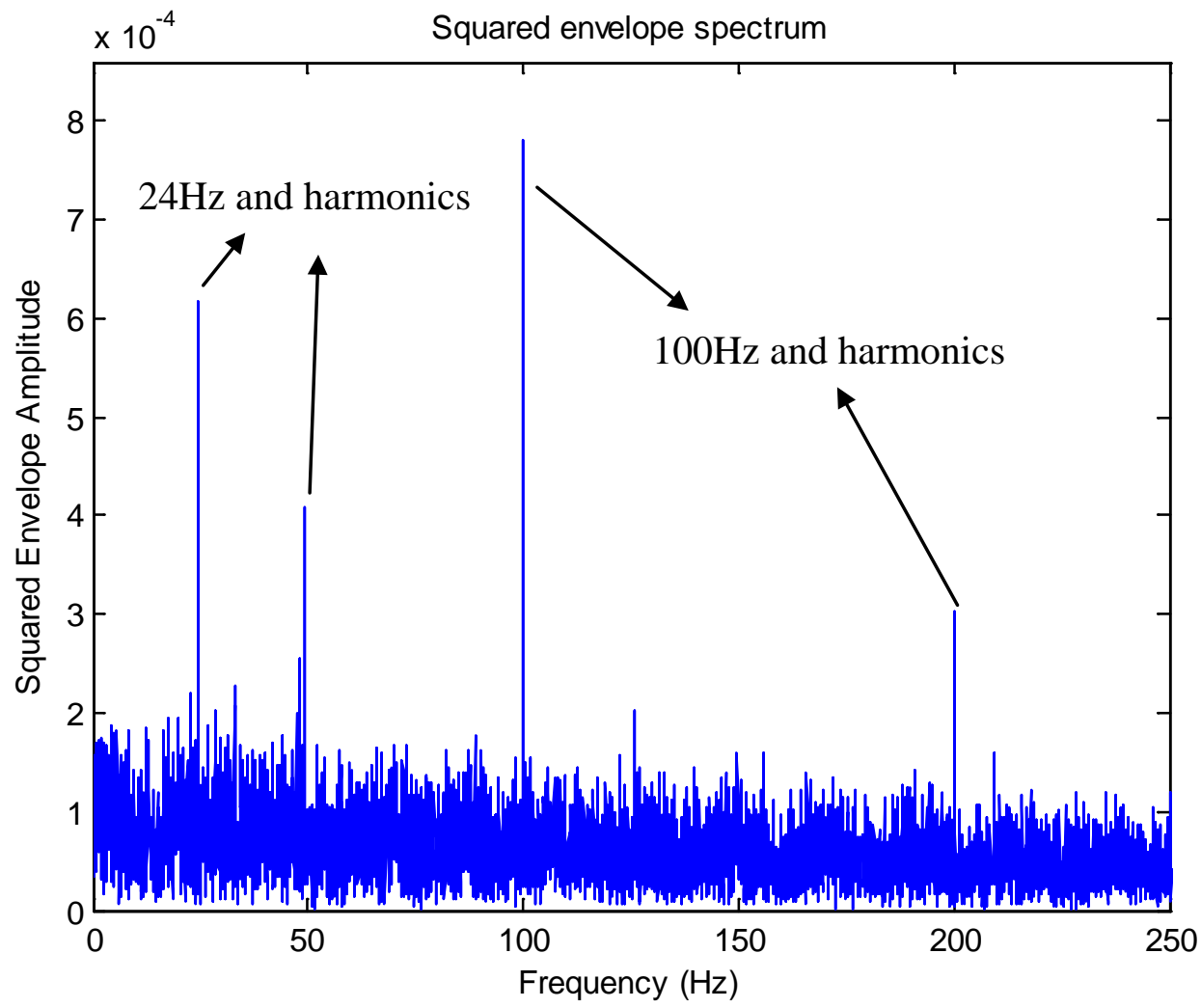

Figure 17 Squared envelope spectrum (Good bearing) 
As a comparison, the resultant optimized SK and squared envelope spectrum by using the external sensor signals are shown in Figure 18 and Figure 19 respectively. It can be seen that the approach by using the external sensor could not detect the inner race fault. A summary of the inner race fault diagnostic performance using external sensor and internal sensor is given in Table 4.

Table 4 Diagnostic results for tests with inner race faults

\begin{tabular}{cccc}
\hline Torque load(Nm) & Sensor location & $\begin{array}{c}\text { Demodulation } \\
\text { band(Hz) }\end{array}$ & $\begin{array}{c}\text { Diagnosis } \\
\text { performance }\end{array}$ \\
\hline 30 & External & $21004-21516$ & Fail \\
\cline { 2 - 4 } & Internal & $44254-44766$ & Success \\
\hline \multirow{2}{*}{50} & External & $10004-10516$ & Fail \\
\cline { 2 - 4 } & Internal & $47404-47916$ & Success \\
\hline 70 & External & $13104-13616$ & Fail \\
\cline { 2 - 4 } & Internal & $46704-47216$ & Success \\
\hline
\end{tabular}

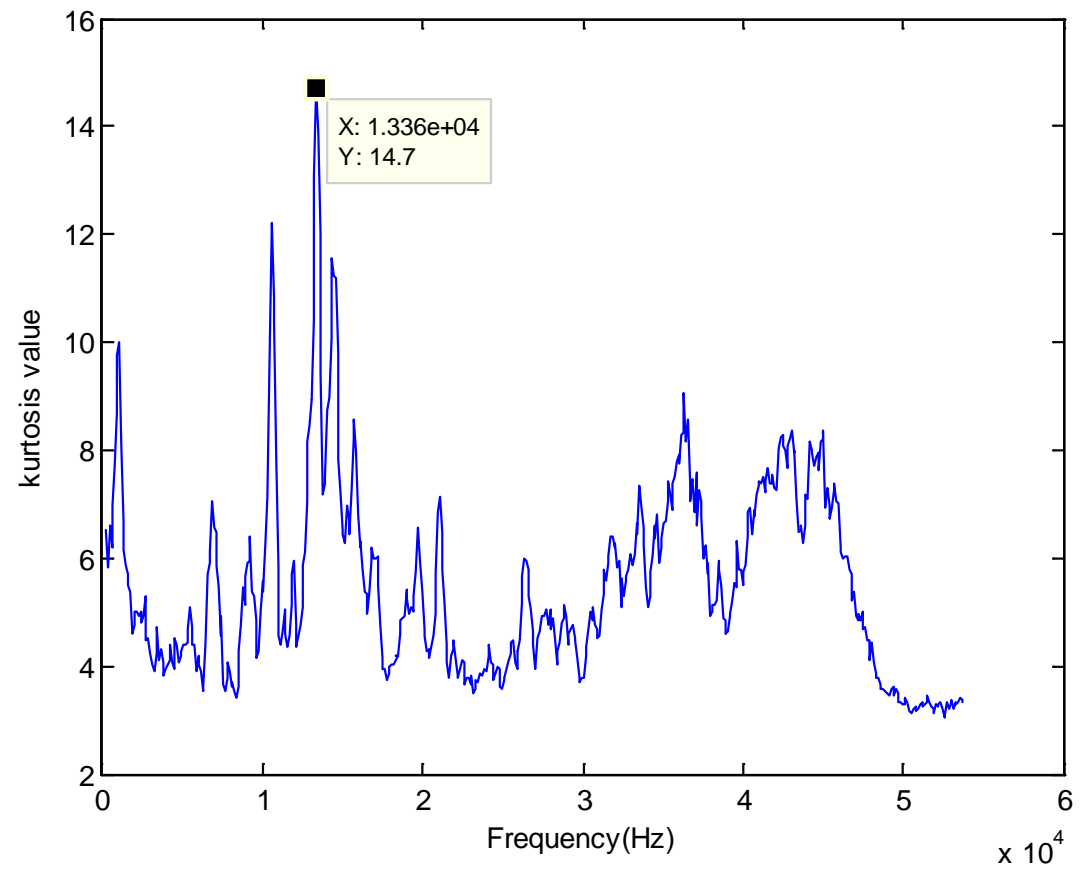

Figure 18 Optimized SK (External sensor) 


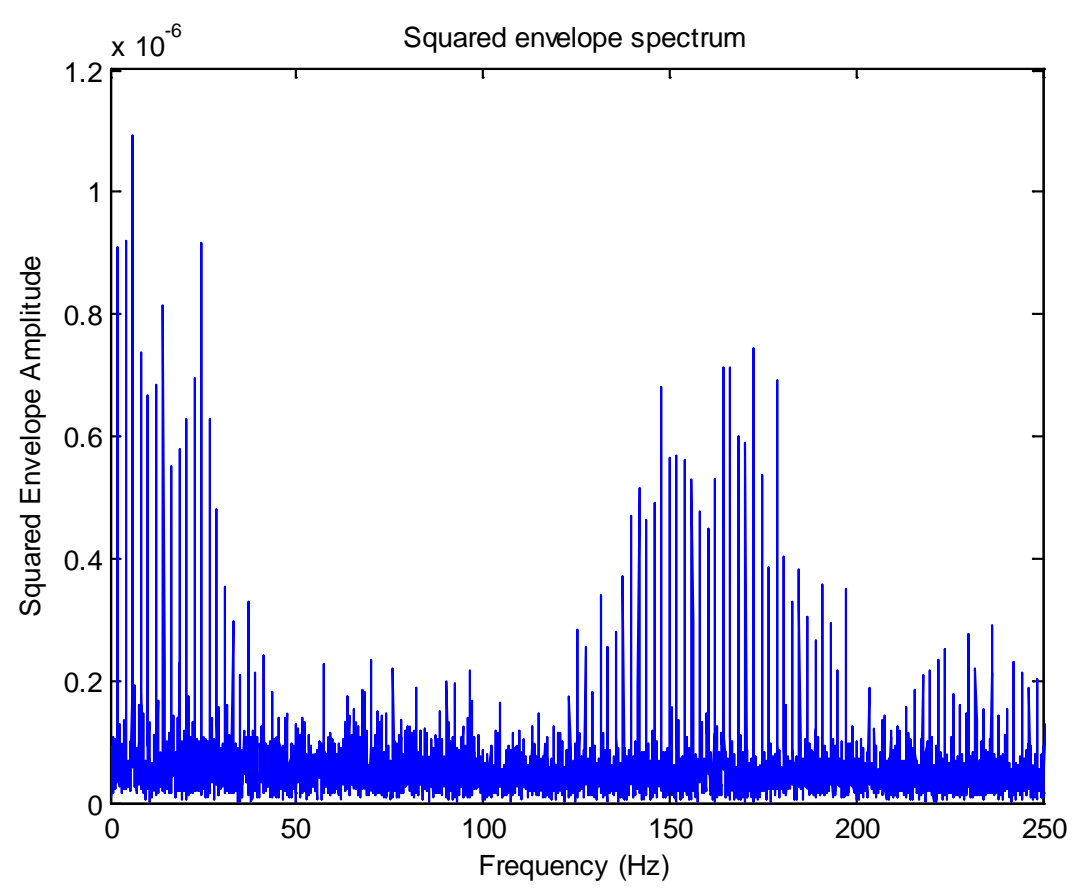

Figure 19 Squared envelope spectrum (External sensor)

\section{Conclusions}

A hybrid approach for fault diagnosis of planetary bearings using an internal vibration sensor and novel signal processing strategies has been introduced in this paper. The internally mounted accelerometer is mounted on and rotates with the planet carrier rather than externally fixed. A set of signal processing methodologies, which combines the techniques of Cepstrum whitening, MED, optimized SK and envelope analysis, is proposed for extracting the fault features in the measured signal through solving the demodulation band selection issue caused by electromagnetic interference and for further optimizing the diagnostic performance of the internal sensor based measurement. The diagnosis performance using the external sensor is also presented for comparison. The results showed that by using the proposed signal processing algorithm, while both the external and internal accelerometer are able to detect the 
outer race faults, the internal sensor has an overwhelmingly superior performance in recognizing the inner race faults in the planet bearings.

\section{Acknowledgement}

The authors would like to thank Mr Andrew Bligh for his contribution on the test rig development, and Dr Wade Smith who produced the data on which the paper is based.

\section{Reference}

[1] Y. Lei, J. Lin, M. J. Zuo, and Z. He, "Condition monitoring and fault diagnosis of planetary gearboxes: A review," Measurement, vol. 48, pp. 292-305, 2014.

[2] R. B. Randall, "Detection and diagnosis of incipient bearing failure in helicopter gearboxes," Engineering Failure Analysis, vol. 11, pp. 177-190, 4// 2004.

[3] P. McFadden and I. Howard, "The detection of seeded faults in an epicyclic gearbox by signal averaging of the vibration," DTIC Document1990.

[4] J. Antoni and R. B. Randall, "Unsupervised noise cancellation for vibration signals: part II-a novel frequency-domain algorithm," Mechanical Systems and Signal Processing, vol. 18, pp. 103-117, 1// 2004.

[5] Z. Feng, M. J. Zuo, J. Qu, T. Tian, and Z. Liu, "Joint amplitude and frequency demodulation analysis based on local mean decomposition for fault diagnosis of planetary gearboxes," Mechanical Systems and Signal Processing, vol. 40, pp. 56-75, 10// 2013.

[6] Z. Feng, M. Liang, Y. Zhang, and S. Hou, "Fault diagnosis for wind turbine planetary gearboxes via demodulation analysis based on ensemble empirical mode decomposition and energy separation," Renewable Energy, vol. 47, pp. 112-126, 11// 2012.

[7] T. Barszcz and R. B. Randall, "Application of spectral kurtosis for detection of a tooth crack in the planetary gear of a wind turbine," Mechanical Systems and Signal Processing, vol. 23, pp. 1352-1365, 5// 2009.

[8] Y. Jing, L. Yip, and V. Makis, "Wavelet analysis with time-synchronous averaging of planetary gearbox vibration data for fault detection, diagnostics, and condition based maintenance," in Mechanical and Electronics Engineering (ICMEE), 2010 2nd International Conference on, 2010, pp. V1-132-V1-136.

[9] Y. Lei, D. Han, J. Lin, and Z. He, "Planetary gearbox fault diagnosis using an adaptive stochastic resonance method," Mechanical Systems and Signal 
Processing, vol. 38, pp. 113-124, 7/5/ 2013.

[10] De Smidt and M. Ryan, "Internal vibration monitoring of a planetary gearbox," University of Pretoria, 2009.

[11] W. Smith, L. Deshpande, R. Randall, and H. Li, "Bearing diagnostics in a planetary gearbox: a study using internal and external vibration signals," 6th International Congress of Condition Monitoring and Diagnostic Engineering Management, Journal of Physics, 11-13 June 2013.

[12] R. B. Randall and N. Sawalhi, "A new method for separating discrete components from a signal," Sound and Vibration, vol. 45, p. 6, 2011.

[13] P. D. McFadden and J. D. Smith, "A Signal Processing Technique for Detecting Local Defects in a Gear from the Signal Average of the Vibration," Proceedings of the Institution of Mechanical Engineers, Part C: Journal of Mechanical Engineering Science, vol. 199, pp. 287-292, October 1, 1985 1985.

[14] J. Antoni and R. B. Randall, "Unsupervised noise cancellation for vibration signals: part I-evaluation of adaptive algorithms," Mechanical Systems and Signal Processing, vol. 18, pp. 89-101, 1// 2004.

[15] P. Borghesani, P. Pennacchi, R. Randall, N. Sawalhi, and R. Ricci, "Application of cepstrum pre-whitening for the diagnosis of bearing faults under variable speed conditions," Mechanical Systems and Signal Processing, 2012.

[16] R. A. Wiggins, "Minimum entropy deconvolution," Geoexploration, vol. 16, pp. 21-35, 4// 1978.

[17] H. Endo and R. B. Randall, "Enhancement of autoregressive model based gear tooth fault detection technique by the use of minimum entropy deconvolution filter," Mechanical Systems and Signal Processing, vol. 21, pp. 906-919, 2// 2007.

[18] N. Sawalhi, R. B. Randall, and H. Endo, "The enhancement of fault detection and diagnosis in rolling element bearings using minimum entropy deconvolution combined with spectral kurtosis," Mechanical Systems and Signal Processing, vol. 21, pp. 2616-2633, 8// 2007.

[19] R. B. Randall and J. Antoni, "Rolling element bearing diagnostics-A tutorial," Mechanical Systems and Signal Processing, vol. 25, pp. 485-520, 2// 2011.

[20] J. Antoni and R. B. Randall, "The spectral kurtosis: application to the vibratory surveillance and diagnostics of rotating machines," Mechanical Systems and Signal Processing, vol. 20, pp. 308-331, 2// 2006.

[21] J. Antoni, "The spectral kurtosis: a useful tool for characterising non-stationary signals," Mechanical Systems and Signal Processing, vol. 20, pp. 282-307, 2// 2006.

[22] J. Antoni, "Fast computation of the kurtogram for the detection of transient faults," Mechanical Systems and Signal Processing, vol. 21, pp. 108-124, 1// 2007.

[23] T. Barszcz and A. JabŁoński, "A novel method for the optimal band selection 
for vibration signal demodulation and comparison with the Kurtogram," Mechanical Systems and Signal Processing, vol. 25, pp. 431-451, 2011.

[24] P. D. McFadden and J. D. Smith, "Vibration monitoring of rolling element bearings by the high-frequency resonance technique — a review," Tribology International, vol. 17, pp. 3-10, 2// 1984.

[25] D. Ho and R. B. Randall, "Optimisation of bearing diagnostic techniques using simulated and actual bearing fault signals," Mechanical Systems and Signal Processing, vol. 14, pp. 763-788, 9// 2000.

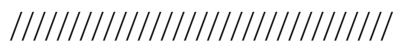

Change to [8]:

J. Yu; V. Makis, "Wavelet analysis with time-synchronous averaging of planetary gearbox vibration data for fault detection and diagnostics," Computer Science and Automation Engineering (CSAE), 2011 IEEE International Conference on , vol.1, no., pp.417,421, 10-12 June 2011. doi: 10.1109/CSAE.2011.5953252 